\title{
On Time Resonances (Explosions) in High-Energy Nuclear Collisions
}

\author{
V.S. Olkhovsky \\ Institute of Nuclear Research of NASU, Kiev, Ukraine \\ Email: olkhovsky@mail.ru
}

Received 28 July 2014; revised 18 September 2014; accepted 22 October 2014

Copyright (C) 2014 by author and OALib.

This work is licensed under the Creative Commons Attribution International License (CC BY).

http://creativecommons.org/licenses/by/4.0/

(c) $\underset{\mathrm{EY}}{\text { (i) }}$ Open Access

\begin{abstract}
The review of the results of time analysis at the range of high-energy nuclear collisions (with the bombarding-particle energies near $0.1-10 \mathrm{GeV} /$ nucleon) is presented. It was shown that the formation of time resonances (explosions) in the decays of compound nuclei or final clots at the range of dense strongly overlapped energy resonances was possible. These time resonances rather well explain a lot of the experimental data.
\end{abstract}

\section{Keywords}

Time Resonance (Explosion) in Compound Nucleus Decay, High-Energy Nuclear Collision

Subject Areas: Modern Physics, Nuclear Physics

\section{Introduction}

In the wide energy region of the bombarding particles more 1 - $10 \mathrm{GeV} /$ nucleon (see, for instance, [1]-[7]) and for the great their number (from $\mathrm{p}$ till ${ }^{20} \mathrm{Ne}$ ), number of targets and of the registered final fragments there are observed the exponentially decreasing inclusive (and sometimes non inclusive) energy spectra without structure. For more heavy bombarding particles such phenomena are observed also smaller energies (see, for instance, [8]). For the analysis of such reactions with heavy ions with energies till $1 \mathrm{GeV} /$ nucleon one can use in a certain degree the fireball model and also the model of intra-nuclear cascade [9] and the model of nuclear fluid [10] works for more high energies in the supposition of the high-dense collision-complexes formation. Between the difficulties of the fireball models there is a problem, why even for high excitations (more than $100 \mathrm{MeV} /$ nucleon) there is formed the statistical equilibrium. In [11], there was proposed other model of "time compound nucleus" for the alternative explanation of high-energy nuclear reactions. This model utilized the preliminary results of eigen states of time operator in the Hamiltonian approach [12]. It was based on the introduction of the formal similarity between the meta-stable states with the eigen complex energies as the eigen states of the Schroedinger equation and the correspondent Fourier transformations with complex eigen values for the equation with time 
operator, canonically conjugate to the Hamiltonian. This model was only the initial step to the time-dependent approach and was not sufficiently justified.

We proposed a new version of the time-evolution approach, starting not only from the principal ideas [13], but also from the known correspondence between the exponential decreasing of behavior of any quantity in any (time or energy) representation and the Lorentzian behavior of its Fourier transformation in the canonically conjugate (i.e. energy or time) representation and then utilizing the results, obtained in [13]-[17] for the properties of compound nuclei in the range of the non-resolved strongly overlapped energy resonances. Here we introduce concretely the phenomenon of time resonance and it is explained the similarity between energy and time resonances. And also there are analyzed the energy and time properties of compound nuclei which are connected with the explosions of time resonances in the evolution decay of final particles.

\section{The Theoretical Description of Time Resonances (Explosions)}

Our theoretical approach is based on [18]-[20]. So far let us choose the reaction amplitude $f_{\alpha \beta}(E)$ and $T$-matrix $T_{\alpha \beta}(E)$ in such forms

$$
f_{\alpha \beta}(E)=C_{\alpha \beta}^{n} \exp \left(-E \tau_{n} / 2 \hbar+i E t_{n} / \hbar\right)
$$

and

$$
T_{\alpha \beta}(E)=T_{\alpha \beta}^{n} \exp \left(-E \tau_{n} / 2+i E t_{n} / \hbar\right)
$$

Here in the certain energy region $E_{\min }<E<\infty$, where $\tau_{n}$ and $t_{n}$ are constants (with the dimension of time), $\tau_{n}$ and $t_{n}$ define the exponential dependence on energy for the corresponding cross section and the linear dependence from energy for the amplitude phase, respectively. $\tilde{T}_{\alpha \beta}^{n}$ is the constant or the very smooth function (inside $\Delta E$ ) on energy $E$ of the final particle. A resonant structure of $\tilde{T}_{\alpha \beta}^{n}$ we so far do not taken evidently into account, supposing it the totally averaged in the limits of the energy spread (or resolution) $\Delta E$, supposing that $\Delta E \ll 2 \hbar / \tau_{n}$.

In this case it is possible to write for the wave packet of final particle in one-dimensional radial asymptotic limit $\Psi_{\beta}\left(z_{\beta}, t\right)=\int_{0}^{\infty} \mathrm{d} E g(E) T_{\alpha \beta}(E) \exp \left[i k z_{\beta}-i E t / \hbar\right]$ the following equation

$$
\Psi_{\beta}\left(R_{\beta}, t\right) \cong \int_{E_{\min }}^{\infty} \mathrm{d} E^{\prime} \exp \left(i k R_{\beta}\right) \exp \left[-E^{\prime} \tau_{n} / 2 \hbar+i E^{\prime}\left(t_{n}-t\right) / \hbar\right]
$$

where $R_{\beta}$ is the radius of interaction in the final channel. Utilizing the simplest rectangular form of $g\left(E^{\prime}\right)$,

$$
g\left(E^{\prime}\right)= \begin{cases}(\Delta E)^{-1 / 2} \exp (i \arg g), & \text { for } E_{\min } \leq E-\Delta E / 2<E^{\prime}<E+\Delta E / 2 \\ 0, & \text { for } E^{\prime}<E-\Delta E / 2 \text { and } E^{\prime}>E+\Delta E / 2\end{cases}
$$

where $\arg g$ is the smooth function of $E$ inside $\Delta E$ we obtain

$$
\begin{aligned}
\Psi_{\beta}\left(R_{\beta}, t\right)= & \frac{\text { const }}{t-t_{n}+i \tau_{n} / 2} \exp \left[E\left(-\tau_{n} / 2+i\left(t_{n}-t\right)\right) / \hbar\right] \\
& \cdot\left[\exp \left[\Delta E\left(-\tau_{n} / 2+i\left(t_{n}-t\right) / 2 \hbar\right)-\exp \left[-\Delta E\left(-\tau_{n} / 2+i\left(t_{n}-t\right) / 2 \hbar\right)\right]\right] .\right.
\end{aligned}
$$

If all energies in the large interval, beginning from $E_{\min }$, are totally filled, i.e.

$$
\left\{\begin{array}{l}
(E+\Delta E / 2) \tau_{n} / 2 \rightarrow \infty \text { and } \\
E-\Delta E / 2 \rightarrow E_{\min }
\end{array}\right.
$$

then we arrive to

$$
\Psi_{\beta}\left(R_{\beta}, t\right)=\frac{\text { const }}{t-t_{n}+i \tau_{n} / 2} \exp \left[E_{\min }\left(-\tau_{n} / 2+i\left(t_{n}-t\right)\right) / \hbar\right] .
$$

It is natural to call such behavior $\Psi_{\beta}\left(R_{\beta}, t\right)$ be time resonance due to the Lorentzian form of factor 
$\frac{1}{t-t_{n}+i \tau_{n} / 2}$ in (6), or explosion (for small values of $\tau_{n}$ ). And inversely, if $\Psi_{\beta}\left(R_{\beta}, t\right)$ has the form (6), the Fourier transformation $\Psi_{\beta}\left(R_{\beta}, t\right)$ will be equal

$$
\int_{-\infty}^{\infty} \mathrm{d} t \Psi_{\beta}\left(R_{\beta}, t\right) \exp (i E t / \hbar)=\text { const } \cdot \exp \left[-E \tau_{n} / 2 \hbar+i E t_{n} / \hbar+E_{\min } \tau_{n} / 2 \hbar\right] .
$$

It is proportional to the amplitude (1). Then we-write (2) in a following way:

$$
\Psi_{\beta}\left(z_{\beta}, t\right)=\int_{E_{\min }}^{\infty} \mathrm{d} E^{\prime} \exp \left(i k R_{\beta}\right) \exp \left[-E^{\prime} \tau_{n} / 2 \hbar+i E^{\prime}\left(t_{n}-t\right) / \hbar\right]
$$
ble

For the small energy spread $(\Delta E \ll E \Delta)$, utilizing the function (3) for $g\left(E^{\prime}\right)$ and introducing a new varia-

$$
y^{\prime}=\sqrt{\frac{i \hbar\left(t-t_{n}-i \tau_{n} / 2\right)}{2 m_{\beta}}}\left[k-\frac{m_{\beta} z_{\beta}}{\hbar\left(t-t_{n}-i \tau_{n} / 2\right)}\right]
$$

we finally obtain:

$$
\Psi_{\beta}\left(R_{\beta}, t\right)= \begin{cases}0, & \text { for } z_{\beta}>v\left(t-t_{n}-t_{i n}^{0}\right) \\ \text { const } \cdot \exp \left[i k r-\frac{i E\left(t-t_{n}-t_{n}^{0}-i \tau_{n} / 2\right)}{\hbar}-\Delta E A(t)\right], & \text { for } z_{\beta} \leq v\left(t-t_{n}-t_{i n}^{0}\right)\end{cases}
$$

where

$$
A(t)=\left[t-t_{n}-t_{n}^{0}-z_{\beta} / v-i \tau_{n} / 2\right] / 2 \hbar
$$

The cross section has the following exponential form:

$$
\sigma_{\alpha \beta}=\left|f_{\alpha \beta}\right|^{2}=\text { const } \cdot \exp \left(-E \tau_{n} / \hbar\right)
$$

When $\tilde{T}_{\alpha \beta}$ or $f_{\alpha \beta}$ has the general form like

$$
f_{\alpha \beta}=\sum_{n=1}^{v} f_{\alpha \beta}^{n} \exp \left[-E \tau_{n} / 2 \hbar+i E t_{n} / \hbar\right]
$$

with several terms $(v=2,3, \cdots)$, the cross section $\sigma_{\alpha \beta}=\left|f_{\alpha \beta}\right|^{2}$ contains not only exponentially decreasing terms, but also oscillating terms with factors $\cos \left[E\left(t_{n}-t_{n^{\prime}}\right) / \hbar\right]$ or $\sin \left[E\left(t_{n}-t_{n^{\prime}}\right) / \hbar\right]$. In the case of 2 terms $(v=2)$ in (2), formula (12) transforms in the following expression

$$
\sigma_{\alpha \beta}=\left|f_{\alpha \beta}^{1}\right|^{2} \exp \left(-E \tau_{1} / \hbar\right)+\left|f_{\alpha \beta}^{2}\right|^{2} \exp \left(-E \tau_{2} / \hbar\right)+2 \operatorname{Re}\left\{f_{\alpha \beta}^{1} f_{\alpha \beta}^{2 \cdot} \exp \left[i E\left(t_{1}-t_{2}\right) / \hbar-E\left(\tau_{1}+\tau_{2}\right) / 2 \hbar\right]\right\}
$$

(where the terms with $\Delta E$ can be neglected, if we suppose that $\Delta E t_{n} \ll E \tau_{n}$ and $\Delta E \tau_{n} \ll E t_{n}$.

The evolution of the survival of the compound nucleus (in the time moment $t$ after its formation) is described by the following function:

$$
L^{c}(t)=1-\int_{t_{0}}^{t} \mathrm{~d} t I(t)
$$

where $I(t)$ is defined by formula [13] [19] $I(t)=\frac{j_{\beta}\left(R_{\beta}, t\right)}{\int_{-\infty}^{\infty} \mathrm{d} t j_{\beta}\left(R_{\beta}, t\right)}$ with the probability flux density

$j_{\beta}\left(z_{\beta}, t\right)=\operatorname{Re}\left[\Psi_{\beta}\left(z_{\beta}, t\right) \frac{i \hbar}{2 m} \frac{\partial \Psi_{\beta}^{\bullet}}{\partial z_{\beta}}\right]$. The initial moment $t_{0}$ current time it is natural to choose in the moment 
$t_{\text {in }}^{0}$ and to suppose that $t_{i n}^{0}=0$. However it is necessary to consider indeterminacy $\delta t=\hbar / \Delta E$ of the duration of the initial wave packet before the collision. Therefore

$$
t_{0} \cong t_{n}^{0}-\delta t=-\delta t=-\hbar / \Delta E
$$

In the region of the time resonance (10) the function $L^{c}(t)$ is essentially non exponential even in the approximation $t_{0}=0$. The qualitative form of $L^{c}(t)$ can be illustrated with help of the strongly simplified examples, utilizing (8) for the very narrow interval near $t=t_{n}$, and also for all the values of $t$, when

$$
j_{\beta}\left(R_{\beta}, t\right)=\operatorname{Re}\left[\Psi_{\beta}\left(R_{\beta}, t\right) \frac{i \hbar}{m_{\beta}} \cdot \lim _{\mathrm{z}_{\beta} \rightarrow R_{\beta}} \frac{\partial \Psi_{\beta}^{\bullet}}{\partial z_{\beta}}\right] \cong \bar{v}\left|\Psi_{\beta}\left(R_{\beta}, t\right)\right|^{2}
$$

where $\bar{v}$ is defined by the integral theorem on the mean value, namely by the expression

$$
\int_{E_{\min }}^{\infty} \mathrm{d} E v A \exp \left(-E \tau_{n} / 2 \hbar\right)=\bar{v} \int_{E_{\min }}^{\infty} \mathrm{d} E A \exp \left(-E \tau_{n} / 2 \hbar\right) .
$$

Then

$$
I(t)=\frac{j_{\beta}\left(R_{\beta}, t\right)}{\int_{-\infty}^{+\infty} \mathrm{d} t j_{\beta}\left(R_{\beta}, t\right)} \cong \frac{\left[\left(t-t_{n}\right)^{2}+\tau_{n}^{2} / 4\right]^{-1}}{\int_{-\infty}^{+\infty} \mathrm{d} t\left[\left(t-t_{n}\right)^{2}+\tau_{n}^{2} / 4\right]^{-1}}=\left(\tau_{n} / 2 \pi\right) \frac{1}{\left(t-t_{n}\right)^{2}+\tau_{n}^{2} / 4}
$$

and

$$
L^{c}(t)=1-\mathrm{d} t I(t)=1-(1 / \pi)[\arctan (y)]_{y=2 t_{0} / \tau_{n}}^{y=2\left(t t_{n}-t_{0}\right) / \tau_{n}} .
$$

Since the curve $\arctan (y)$ has the form, depicted in Figure 1, in the case $2 t_{0} / \tau_{n} \rightarrow-\infty$ (the quantity $\tau_{n}$ is small) the function $L^{c}(t)$ has the form, depicted in Figure 2 (the curve 1).

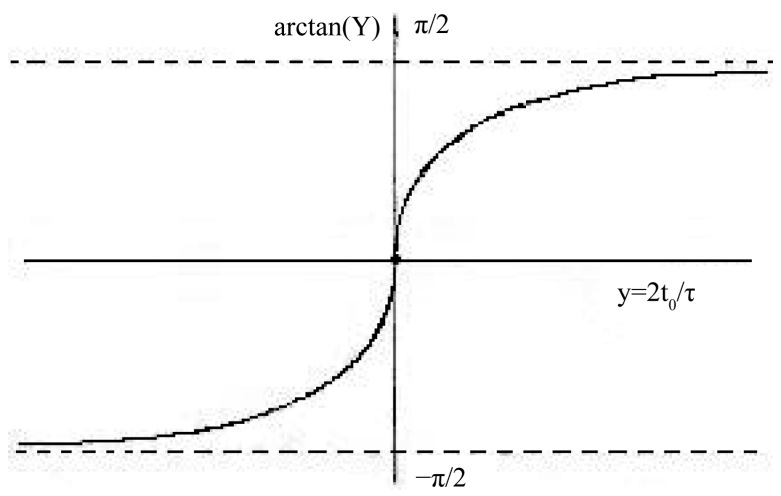

Figure 1. The function $\arctan (y)$ for $2 t_{0} / \tau_{n} \rightarrow-\infty$.

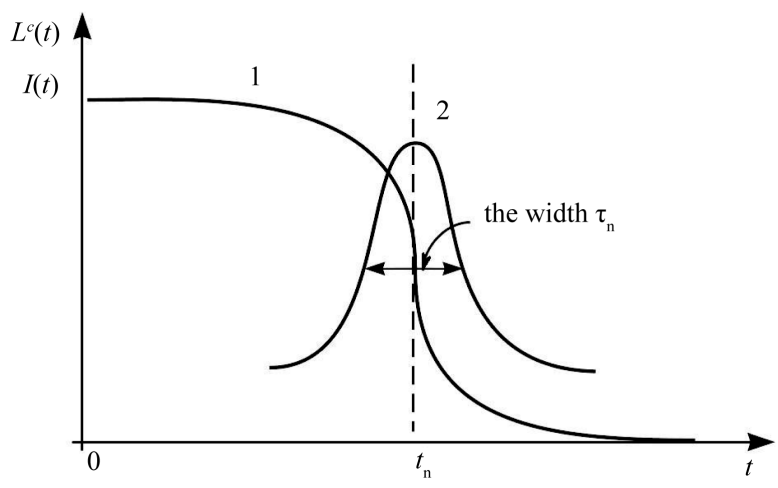

Figure 2. $L^{c}(t)$ (the curve 1) and $I(t)$ (the curve 2). 
In this case

$$
L^{c}(t)=1-\pi^{-1}\left[\arctan \left(2\left(t-t_{n}-t_{0}\right) / \tau_{n}\right)+\pi / 2\right]
$$

and

$$
L^{c}(t)= \begin{cases}1, & \text { when } 0 \leq t<t_{n}=0\left(c-2 t_{0} / \tau_{n} \rightarrow \infty\right) \text { and } \\ 0, & \text { when } t \rightarrow \infty .\end{cases}
$$

From the simple form of Figure 2, it is easy to see that $t_{n}$ can be interpreted as the Poincare' period of internal motion of the compound nucleus (after its formation and before its decay), when $t_{n} \gg \tau_{n}$. Such behavior of $L^{c}(t)$ was studied in [19] [20].

If we precisely consider the compound-resonance structure of $T_{\alpha \beta}$, then the strongly non exponential form of $L^{c}(t)$ and $I(t)$ will take place, as it is depicted in Figure 2, for the strong overlapping of the energy resonances, when

$$
\Gamma_{J S \Pi} \ll N_{J S \Pi} / 2 \pi \rho_{J S \Pi}
$$

( $\Gamma_{J S \Pi}$ and $\rho_{J S \Pi}$ are the mean resonance width and level density, $N_{J S \Pi}$ is the number of open channels, JSח are the values of the total momentum, spin and parity, respectively). The small probability of the compound-nucleus decay for $t<t_{n}$ (inside the Poincare' cycle) can be explained by the consequence of the multiply meta-stable states in the region of the overlapped energy resonances. In the case of several time resonances it can signify the superposition of several strongly overlapped groups of energy resonances with different values of JSח in the same compound nucleus or the formation of several compound nuclei with the different numbers of participating nucleons.

In particular, for the inclusive energy spectra of the $k$-th final fragment it is possible to use the following expression

$$
\begin{aligned}
\sigma_{i n c, k}\left(E_{k}\right) & =\sum_{n=1}^{2}\left|C_{n} \exp \left[\left(i t_{n}-\tau_{n} / 2\right) E_{k} / \hbar\right]\right|^{2} \\
& =\sum_{n=1}^{2}\left|C_{n}\right|^{2} \exp \left(-E_{k} \tau_{n} / \hbar\right)+2 \operatorname{Re} C_{1}^{*} C_{2} \exp \left\{\left[i\left(t_{2}-t_{1}\right)-\left(\tau_{1}+\tau_{2}\right) / 2\right] E_{k} / \hbar\right\} .
\end{aligned}
$$

\section{The Comparison with the Experimental Data}

For the analysis of the observed experimental spectra of a single final fragment it is necessary to sum (or average) the expressions like (13) or (21) over the subfamilies of the final states (with various quantum numbers JSח, where J, L, S and $\Pi$ are quantum numbers of the total momentum, orbital momentum, spin and parity, respectively) and channels, sometimes coherently and sometimes incoherently. And for inclusive energy spectrum of $k$-th final fragment we will use the expression (21).

In Figures 3-6 are represented some calculated inclusive energy spectra $\sigma_{i n c, k}\left(E_{k}\right)$ in the semi-logarithmic scale in compare with the experimental data from [8] [10] [11].

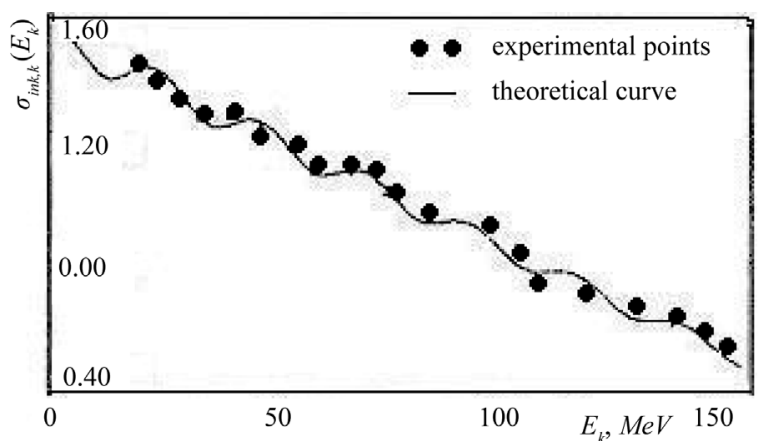

(a)

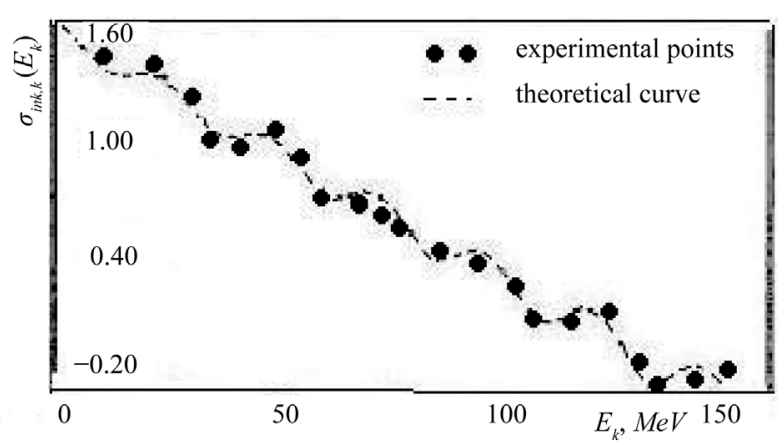

(b)

Figure 3. The inclusive process $\mathrm{p}+\mathrm{C} \rightarrow{ }^{7} \mathrm{Be}+\mathrm{X}$ (protons of $2.1 \mathrm{GeV}$ ), experimental data are taken from [10]. (a) $C_{1}=0.04$, $C_{2}=0.36\left(\theta=90^{\circ}\right) ;(b) C_{1}=0.35, C_{2}=0.05\left(\theta=160^{\circ}\right)$. 


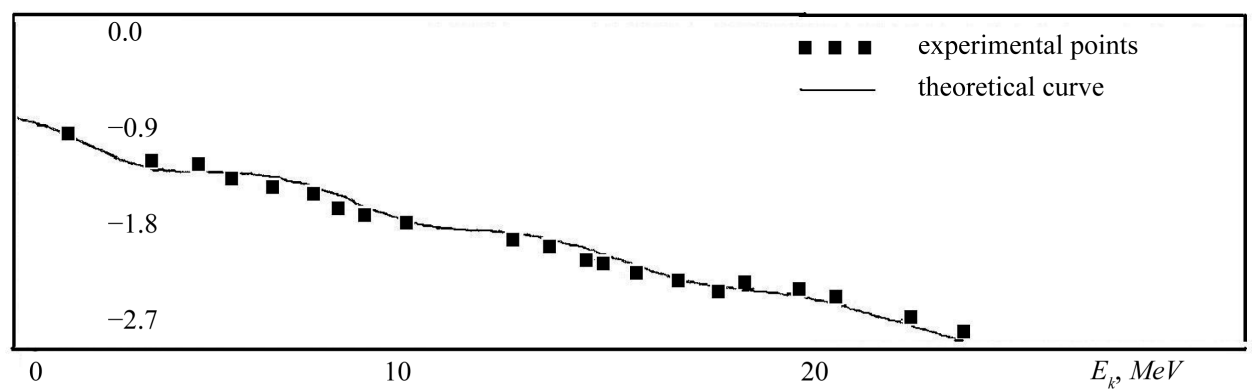

(a)

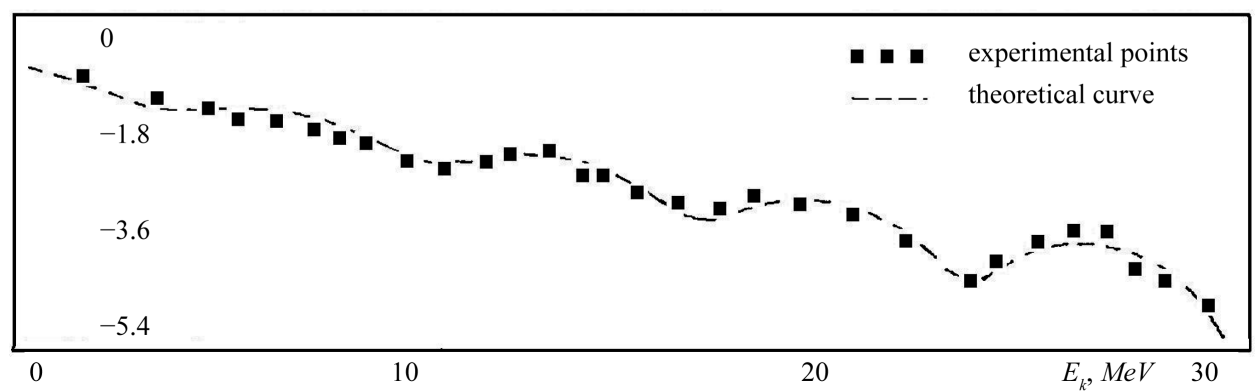

(b)

Figure 4. The inclusive process ${ }^{4} \mathrm{He}+\mathrm{Ta} \rightarrow \mathrm{t}+\mathrm{X}$ (720 MeV/nucleon), experimental data are taken from [11]. (a) $C_{1}=0.18, C_{2}=1.02\left(\theta=60^{\circ}\right)$; (b) $C_{1}=1.13, C_{2}=0.07\left(\theta=90^{\circ}\right)$.

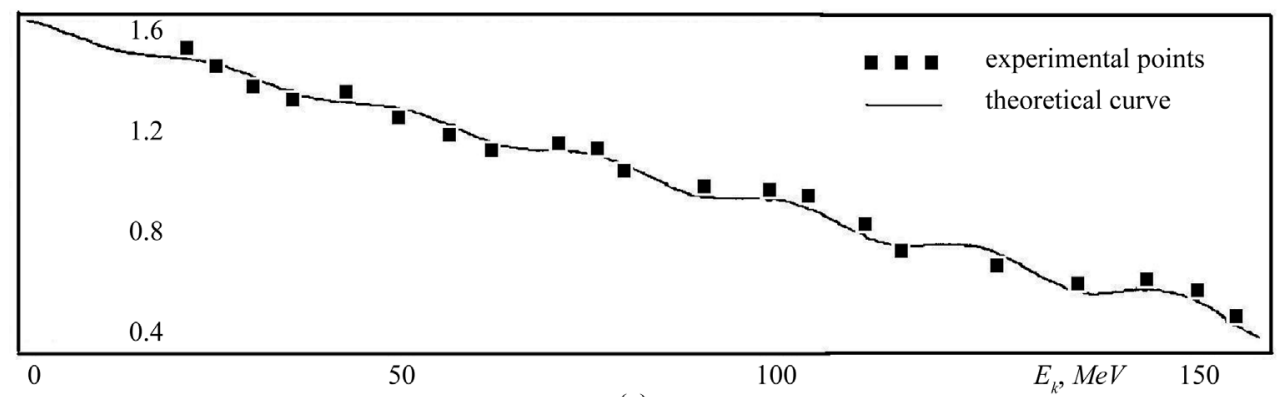

(a)

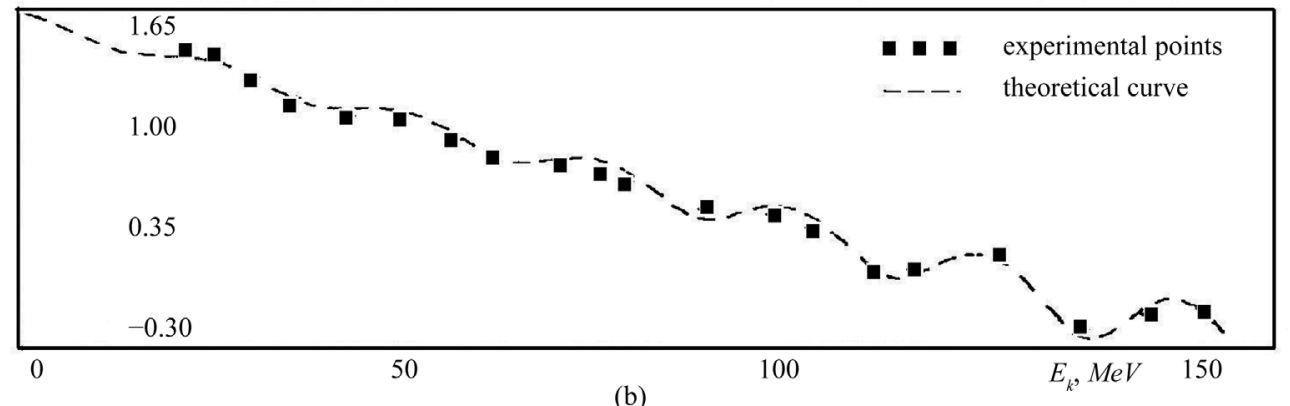

(b)

Figure 5. The inclusive process ${ }^{20} \mathrm{Ne}+\mathrm{U} \rightarrow \mathrm{p}+\mathrm{X}$ (1045 MeV/nucleon), experimental data are taken from [11]. (a) $C_{1}=0.35, C_{2}=5.65\left(\theta=90^{\circ}\right)$; (b) $C_{1}=5.65, C_{2}=0.35\left(\theta=150^{\circ}\right)$.

In Figures 3-6, $\theta$ is the detected angle of $k$-th fragment in emission. The values of $\tau_{1}, \tau_{2}$ and $t_{2}-t_{1}$, which were found in [17] from the fitting of theoretical curves to the experimental data, are written in Table 1.

Since the inclination of energy spectra is essentially increases with the angle increasing, it signifies that the increasing contribution of the compound-nucleus states with larger values of $t_{n}$ and $\tau_{n}$ is connected with the formation of more heavy compound nuclei at the lesser velocity in $L$-system. It agrees with the observed in [9] [11] [19] phenomena of more clear oscillations for the intermediate emission angles. 


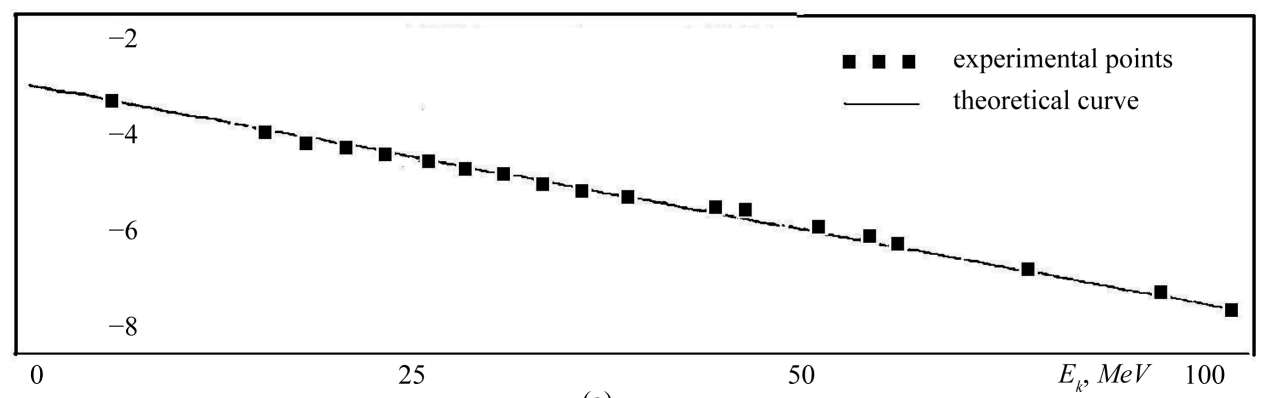

(a)

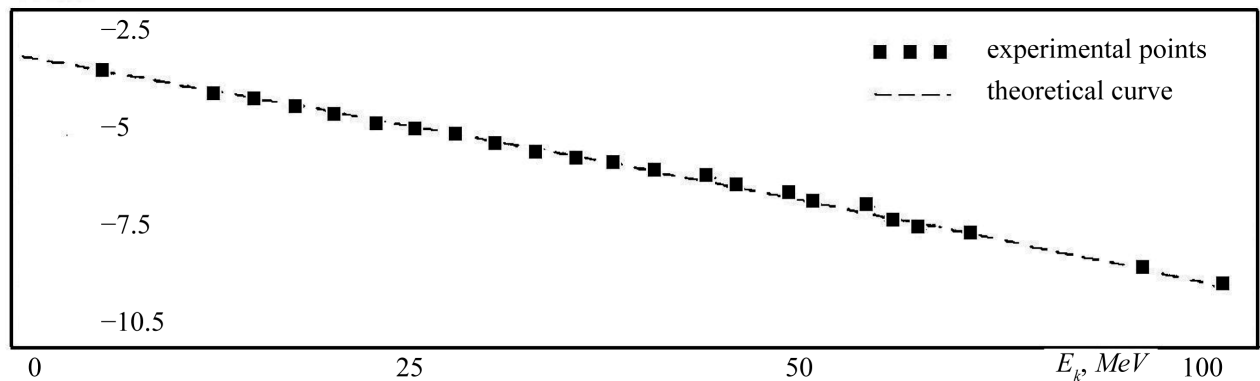

(b)

Figure 6. The inclusive process ${ }^{40} \mathrm{Ar}+{ }^{51} \mathrm{~V} \rightarrow \mathrm{p}+\mathrm{X}$ (41 MeV/nucleon); experimental data are taken from [8]. (a) $C_{1}=0.002, C_{2}=0.03\left(\theta=97^{\circ}\right)$; (b) $C_{1}=0.03, C_{2}=0.022\left(\theta=129^{\circ}\right)$.

Table 1. Parameters of time resonances for some inclusive spectra.

\begin{tabular}{ccccc}
\hline Reaction & $\begin{array}{c}\text { Energy of bomb. particle } \\
\text { GeV/nucleon }\end{array}$ & $\tau_{1}, 10^{-23}$ sec & $\tau_{2}, 10^{-23}$ sec & $t_{2}-t_{1}, 10^{-22}$ sec \\
\hline $\mathrm{P}+\mathrm{C} \rightarrow{ }^{7} \mathrm{Be}+\mathrm{X}$ & 2.1 & 10.45 & 17.0 & 5.95 \\
${ }^{20} \mathrm{Ne}+\mathrm{Al} \rightarrow \mathrm{p}+\mathrm{X}$ & 0.393 & 0.1 & 0.99 & 1.7 \\
${ }^{4} \mathrm{He}+\mathrm{Ta} \rightarrow \mathrm{t}+\mathrm{X}$ & 0.72 & 1.72 & 3.15 & 1.22 \\
${ }^{20} \mathrm{Ne}+\mathrm{U} \rightarrow \mathrm{p}+\mathrm{X}$ & 1.045 & 0.92 & 1.7 & 1.72 \\
${ }^{20} \mathrm{Ar}+\mathrm{V} \rightarrow \mathrm{p}+\mathrm{X}$ & 0.041 & 7.5 & 9.0 & 0.20 \\
${ }^{132} \mathrm{Xe}+\mathrm{Au} \rightarrow \mathrm{p}+\mathrm{X}$ & 0.044 & 6.0 & 7.0 & 1.0 \\
${ }^{20} \mathrm{Ne}+\mathrm{U} \rightarrow \mathrm{p}+\mathrm{X}$ & 0.4 & 1.7 & 2.2 & 0.10 \\
${ }^{20} \mathrm{Ne}+\mathrm{U} \rightarrow \mathrm{d}+\mathrm{X}$ & 0.25 & 4.2 & 7.2 & 0.10 \\
\hline
\end{tabular}

It is possible that for the most easy compound system $(p+C)$, represented here, there is a superposition of the direct process (i.e. $n=0$ instead of $n=1$ ) and the time resonance $(n=2)$, since the difference $t_{2}-t_{1(0)}$ is noticeably larger than usually.

Later there were performed new calculations and their comparison with the experimental data. They are represented in Figure 7, Figure 8.

The values of $\tau_{1}, \tau_{2}$ and $t_{2}-t_{1}$ in sec, which were found in [20] from the agreement of theoretical curves with the experimental data, are represented in Table 2 for Figure 7 and in Table 3 for Figure 8, respectively.

\section{The Explanation of the Time-Resonances Structure in the Cross Sections of High-Energy Nuclear Reactions in the Region of the Densely Situated Strongly Overlapped Energy Resonances}

How is it possible to explain the manipulations with relatively smooth energy behavior of the expressions (11) and (13) for the cross sections or the expressions (1) and (12) for $T_{\alpha \beta}$ or $f_{\alpha \beta}$, which correspond to time resonances and simultaneously to the experimental data on cross sections, although really the amplitudes have to fluctuate strongly with energy in the region of strongly overlapped energy resonances for extremely high energies? 


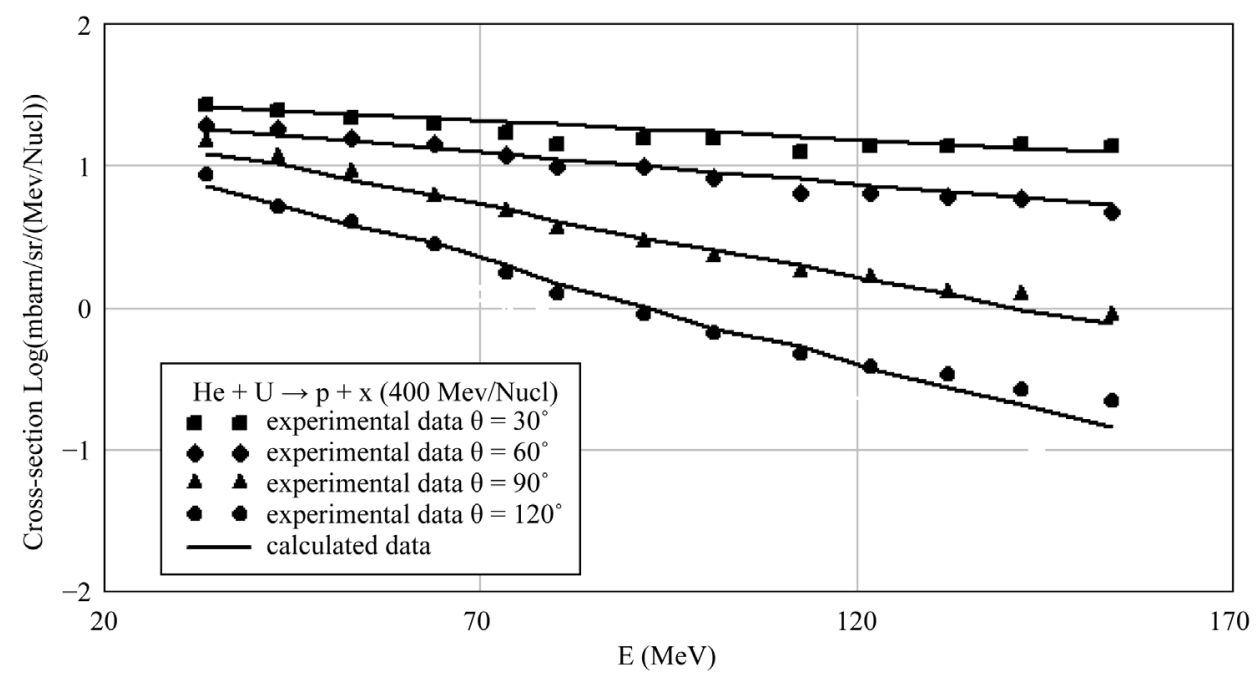

Figure 7. Inclusive energy spectrum of ${ }^{4} \mathrm{He}+\mathrm{U} \rightarrow \mathrm{p}+\mathrm{X}$, of $400 \mathrm{MeV} /$ nucleon.

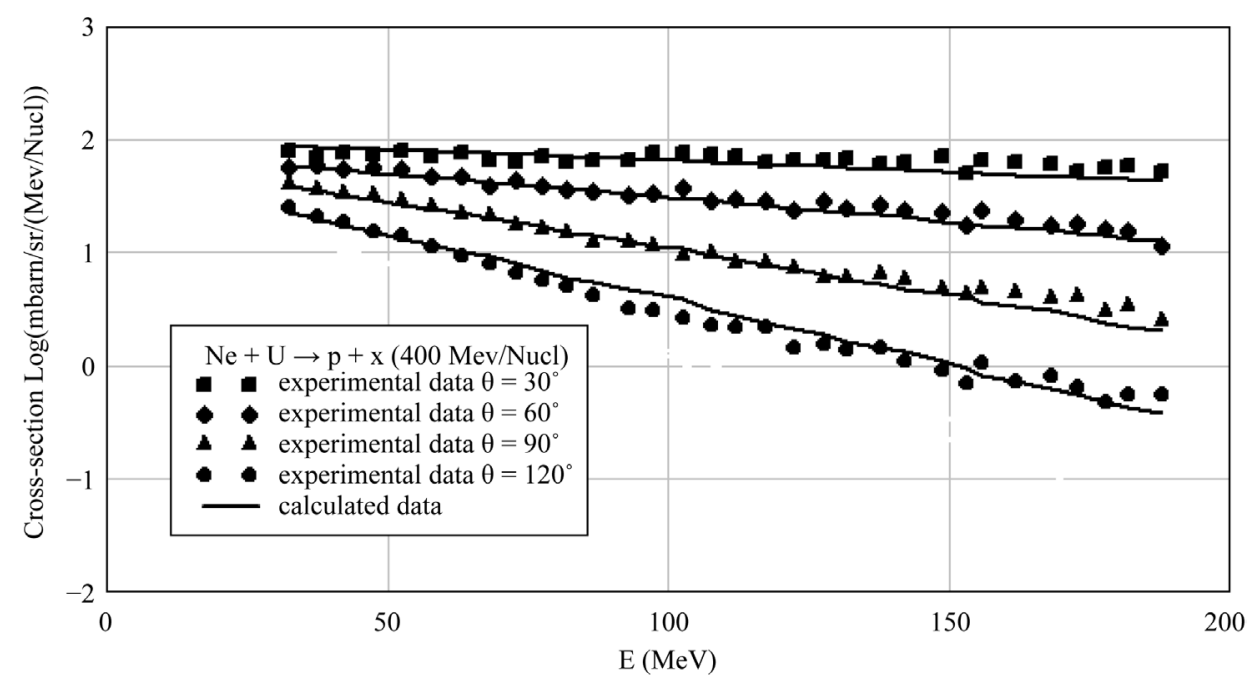

Figure 8. Inclusive energy spectrum of ${ }^{20} \mathrm{Ne}+\mathrm{U} \rightarrow \mathrm{p}+\mathrm{X}$, of $400 \mathrm{MeV} /$ nucleon.

Table 2. The parameters for time resonances in the case of the inclusive spectrum of ${ }^{4} \mathrm{He}+\mathrm{U} \rightarrow \mathrm{p}+\mathrm{X}$, of $400 \mathrm{MeV} / \mathrm{nucleon}$ for different angles $\theta$.

\begin{tabular}{cccccc}
\hline$\theta$ & $\tau_{1}\left(10^{-23} \mathrm{~s}\right)$ & $\tau_{2}\left(10^{-23} \mathrm{~s}\right)$ & $t_{2}-t_{1}\left(10^{-23} \mathrm{~s}\right)$ & $C_{1}$ & $C_{2}$ \\
$30^{\circ}$ & 0.38 & 0.38 & 0.25 & 2.8 & 2.8 \\
$60^{\circ}$ & 0.64 & 0.64 & 0.25 & 2.6 & 2.6 \\
$90^{\circ}$ & 1.5 & 1.5 & 0.25 & 2.5 & 2.5 \\
$120^{\circ}$ & 2.1 & 2.1 & 0.25 & 2.3 & 2.3 \\
\hline
\end{tabular}

At first sight, in the region of high energies the structure of energy resonances has to vanish not only due to the “smoothing” by energy spreads (since $\Delta E \gg \Gamma_{J S \Pi},\left(\rho_{J S \Pi}\right)^{-1}$ ), but also de facto due to the strong decreasing of the probability of the formation of the intermediate long-living many-nucleon states. The density of the compound-resonances is quickly increases, beginning from the low-energy well resolved energy resonances where the various versions of the Fermi-gas model with the shell-model and collective-model corrections work rather successfully work. Only near $30-40 \mathrm{MeV} /$ nucleon in the compound system it is possible to expect the 
Table 3. The parameters for time resonances in the case of the inclusive spectrum of ${ }^{20} \mathrm{Ne}+\mathrm{U} \rightarrow \mathrm{P}+\mathrm{X}$, of $400 \mathrm{MeV} / \mathrm{nucleon}$ for different angles $\theta$.

\begin{tabular}{cccccc}
\hline$\theta$ & $\tau_{1}\left(10^{-23} \mathrm{~s}\right)$ & $\tau_{2}\left(10^{-23} \mathrm{~s}\right)$ & $t_{2}-t_{1}\left(10^{-23} \mathrm{~s}\right)$ & $C_{1}$ & $C_{2}$ \\
\hline $30^{\circ}$ & 0.25 & 0.25 & 0.25 & 5 & 5 \\
$60^{\circ}$ & 0.6 & 0.6 & 0.25 & 4.5 & 4.5 \\
$90^{\circ}$ & 1.2 & 1.2 & 0.25 & 4.2 & 4.2 \\
$120^{\circ}$ & 1.7 & 1.7 & 0.25 & 3.6 & 3.6 \\
\hline
\end{tabular}

saturation effects and the further strong decreasing of the densities. However namely for these energies the resonances of another structure can appear. These resonances are connected with the local excitations of longliving intermediate many-quark-gluon states of the baryon subsystems (see [21]).

Let us consider the possibility of the abovementioned explanation of the structure of time resonances more attentively, limited ourselves only by the partial JSח-amplitudes $T_{\alpha \beta}^{J S \Pi}=\delta_{\alpha \beta}-S_{\alpha \beta}^{J S \Pi}$, where $S_{\alpha \beta}^{J S \Pi}$ is the element of the $S$-matrix.

As it was said above, for the sufficiently high energies if we neglect bound and virtual states and the threshold particularities we can describe the S-matrix by the many-channel S-matrix [22] and for the simplest Baz'-Newton conditions (see [23]) this many-channel S-matrix

$$
\hat{S}^{(J)}(E)=\hat{U}^{(J)} \prod_{v=1}^{N}\left(1-\frac{i \Gamma_{v}^{(J)}}{E-E_{v}^{(J)}+i \Gamma_{v}^{(J)} / 2}\right) \hat{U}^{(J) \mathrm{T}},
$$

where the unitary matrix $\hat{U}^{(J)}$ and the projection matrixes $\hat{P}_{v}^{(J)}\left(\hat{P}_{v}^{(J)}=\hat{P}_{v}^{(J) \bullet}=\hat{P}_{v}^{(J) 2}\right.$, Trace $\left.\hat{P}_{v}^{(J)}=1\right)$ are practically do not depend on energy) acquires a such form:

$$
\hat{S}=\hat{S}_{b}-\hat{a}\left(1-\prod_{n} \frac{\varepsilon-\varepsilon_{n}-i \Gamma_{n} / 2}{\varepsilon-\varepsilon_{n}+i \Gamma_{n} / 2}\right)
$$
where $\hat{S}_{b}=\hat{U} \hat{U}^{\mathrm{T}}$ and $\hat{a}=\hat{U}\langle\hat{P}\rangle \hat{U}^{\mathrm{T}}$. The averaged on energy the $S$-matrix $\langle\hat{S}\rangle_{\Delta \varepsilon}$ in this case in accordance
with [22]:

$$
\langle\hat{S}\rangle_{\Delta E}=\hat{S}_{b}-\hat{a}[1-\exp (-\pi \Gamma / \rho)]
$$

for unresolved resonances in $\left(\Delta E \gg \rho^{-1}, \Gamma\right.$ ) and the fluctuating $S$-matrix $\hat{S}^{c}$ (or $S$-matrix of the compound nucleus) is equal

$$
\hat{S}^{c}=S-\langle S\rangle_{\Delta \varepsilon}=\hat{a}\left[\prod_{n} \frac{\varepsilon-\varepsilon_{n}-i \Gamma_{n} / 2}{\varepsilon-\varepsilon_{n}+i \Gamma_{n} / 2}-\exp (-\pi \Gamma \rho)\right]
$$

We repeat that $\hat{S}_{b}$ and $\hat{a}$ almost do not depend on energy (slowly change with energy). For the strongly overlapped resonances when $\pi \Gamma \rho \gg 1$.

$$
\hat{S}^{c} \rightarrow \hat{a} \prod_{n}\left(\frac{\varepsilon-\varepsilon_{n}-i \Gamma_{n} / 2}{\varepsilon-\varepsilon_{n}+i \Gamma_{n} / 2}\right)
$$

and the averaged over energy cross section of the processes, going through the step of formation of compound nucleus $\left\langle\sigma_{\alpha \beta}^{c}\right\rangle_{\Delta \varepsilon}$, is evidently proportional to $\left|a_{\alpha \beta}\right|^{2}$ :

$$
\left\langle\sigma_{\alpha \beta}^{c}\right\rangle \sim\left\langle\left|S_{\alpha \beta}^{c}\right|^{2}\right\rangle_{\Delta \varepsilon}=\left|a_{\alpha \beta}\right|^{2}
$$

(here and below we continue to omit the indexes JSח). If the initial energy of bombarding particles is fixed and therefore the total energy $\varepsilon$ is also fixed (to within $\Delta \varepsilon$ ), the cross section (24) can be re-written in the form

$$
\left\langle\sigma_{\alpha \beta}^{c}\right\rangle_{\Delta E} \sim\left\langle\left|S_{\alpha \beta}^{c}\right|^{2}\right\rangle_{\Delta \varepsilon} \cong\left|a_{\alpha \beta}\right|^{2},
$$

where $\Delta E$ is defined by $\Delta \varepsilon$ and the energy resolution of the detector of final fragments. 
From [13] [19]) one can see that the averaged over energy time delay of compound nucleus and the variance of the time-delay-of-compound-nucleus distributions are defined by such general relations

$$
\left\langle\tau_{\alpha \beta}^{c}\right\rangle=\left\langle\left|S_{\alpha \beta}^{c}\right|^{2} \frac{\hbar \partial \arg S_{\alpha \beta}^{c}}{\partial E}\right\rangle /\left\langle\left|S_{\alpha \beta}^{c}\right|^{2}\right\rangle
$$

and

$$
D \tau_{\alpha \beta}^{c}=\frac{\hbar^{2}\left\langle\left(\partial\left|S_{\alpha \beta}^{c}\right| / \partial E\right)^{2}\right\rangle_{\Delta E}}{\left\langle\left|S_{\alpha \beta}^{c}\right|^{2}\right\rangle_{\Delta E}}+\frac{\hbar^{2}\left\langle\left|S_{\alpha \beta}^{c}\right|^{2}\left(\partial \arg S_{\alpha \beta}^{c} / \partial E\right)^{2}\right\rangle_{\Delta E}}{\left\langle\left|S_{\alpha \beta}^{c}\right|^{2}\right\rangle_{\Delta E}}-\left\langle\tau_{\alpha \beta}^{c}\right\rangle^{2},
$$

respectively (energy $E$ is the kinetic energy of final fragment). From (23a) and (25) one can see (see also [13] [19]) that the mean time delay, averaged over all channels, is equal

$$
\left\langle\tau^{c}\right\rangle=\left\langle\sum_{n} \frac{\hbar \Gamma}{\left(\varepsilon-\varepsilon_{n}\right)^{2}+\Gamma_{n}^{2} / 4}\right\rangle_{\Delta E}=2 \pi \hbar \rho
$$

in the approximation of continuum ( $\sum_{n} \rightarrow \int \rho \mathrm{d} \varepsilon$ for the quantities, averaged over energy). And $D \tau_{\alpha \beta}^{c}$ in the same continuum approximation

if

$$
D \tau_{\alpha \beta}^{c}=\frac{\hbar^{2}\left\langle\left(\partial\left|a_{\alpha \beta}\right| / \partial E\right)^{2}\right\rangle_{\Delta E}}{\left\langle\left|a_{\alpha \beta}\right|^{2}\right\rangle_{\Delta E}}
$$

$$
\left\langle\tau^{c}\right\rangle^{2}, \exp (-\pi \rho \Gamma) \ll \frac{\hbar^{2}\left\langle\left(\partial\left|a_{\alpha \beta}\right| / \partial E\right)^{2}\right\rangle_{\Delta E}}{\left\langle\left|a_{\alpha \beta}\right|^{2}\right\rangle_{\Delta E}}
$$

Now it is possible to see the mathematical similarity (even coincidence) between the cross section of compound nucleus (24) under above-mentioned conditions (the Baz'-Newton condition and for the strong resonance overlapping when $\pi \Gamma \rho \gg 1$ ) and the time-resonance cross section (15) for a short time resonance. Therefore, returning to the expression $T_{\alpha \beta}=\delta_{\alpha \beta}-S_{\alpha \beta}$ with $S_{\alpha \beta} \rightarrow S_{\alpha \phi}^{c}$, defined by (23a) for strongly overlapped resonances (with $\pi \Gamma \rho \gg 1$ ), we can re-write (28) approximately in such form

$$
\sigma_{\alpha \chi}^{c(n)} \sim\left|a_{\alpha \beta}^{(n)}\right|^{2} \sim \exp \left(-E \tau_{n} / \hbar\right)
$$

(if $\hbar / \tau_{n}>\Delta E$ for small $\Delta E$ ). And under the same conditions

$$
D \tau^{c} \cong\left(\tau_{n}\right)^{2} / 4
$$

(here and further we write $D \tau^{c}$ without indexes $\alpha \beta$ ).

If $\tau_{n} \ll 2 \pi \hbar \rho$ (it is possible when $\Delta E \gg \rho^{-1}$ ), then $D \tau_{c} \cong\left(\tau_{n}\right)^{2} / 4 \ll\left(\left\langle\tau^{c}\right\rangle\right)^{2}$ and we have a narrow time resonance (explosion) of the compound nucleus.

When there are some independent non-fluctuating projectors $\hat{P}_{v}=\left\langle\hat{P}^{(v)}\right\rangle, v=1,2, \cdots, \eta \quad(\eta$ is much lesser of the resonance number), it is possible to obtain at the same reasoning the result like (21) for $\sigma_{\alpha \beta}$ with oscillating terms.

Under more realistic Lyuboshitz conditions of the statistically equivalent channels of the compound-nucleus decay [14]-[16] (see also [13] [19]), when the fluctuations of $\hat{P}_{n}$ are the same in all open channels, it is possible to show that

$$
\left\langle\tau^{c}\right\rangle=2 \pi \hbar \rho / N T
$$


where $T=1-\exp (-2 \pi \rho \Gamma / N)$ and the sum of last two terms in the right part of relation (26) for $D \tau^{c}$ can be neglected in the continuum approximation. From (32) it is clear that for strongly overlapped resonances when $\pi \rho \Gamma / N \gg 1$ and $T \rightarrow 1$, we have:

$$
\left\langle\tau^{c}\right\rangle=2 \pi \hbar \rho / N
$$

In [13] [19] it was shown that under the same conditions and when $S_{b}$ can be considered as independent from energy $\varepsilon$ (and $E$ ), $D \tau^{c} \ll\left\langle\tau^{c}\right\rangle^{2}$. If then one extend the Hauser-Feshbach formula for the compoundnuclear-reactions cross sections $\left\langle\sigma_{\alpha \beta}^{c}\right\rangle$ into the region of high energies, then under the same conditions it is possible to be easily convinced in such behavior of $\left\langle\sigma_{\alpha \beta}^{c}\right\rangle \cong N^{-1} \cong \exp \left(-E \tau_{n} / \hbar\right)$. Under the Lyuboshitz conditions for the strongly overlapped resonances, $D \tau^{c} \cong\left(\tau_{n}\right)^{2} / 4 \ll\left\langle\tau^{c}\right\rangle^{2}$-and the exponential decreasing of energy spectra of final fragments corresponds to the narrow time resonance (explosion) of the compound nucleus. There are possible also the cases when we can observe either one, or several time resonances (explosions) in cross sections.

\section{Conclusion}

The review of the results of papers [6]-[20], dedicated to time resonances (explosions) with utilization of the theoretic researches in [18]-[20] in the Simonius representation of the $S$-matrix [22], had been made. With rather simple assumptions it had been shown that in the region of the very dense overlapping resonances, the phenomenon of time resonances (explosions) can well explain the experimental data for high-energy nuclear collisions even with the presence of slight oscillations in inclusive energy spectra.

\section{References}

[1] Gosset, J., Gutbrod, H., Meyer, W., et al. (1977) Central Collisions of Relativistic Heavy Ions. Physical Review C, 16, 629. http://dx.doi.org/10.1103/PhysRevC.16.629

[2] Westfall, G., Sexstro, R., Poskanzer, A., et al. (1978) Energy Spectra of Nuclear Fragments Produced by High Energy Protons. Physical Review C, 17, 1368. http://dx.doi.org/10.1103/PhysRevC.17.1368

[3] Cumming, J., Haustein, P., Ruth, T., et al. (1978) Spallation of Copper by 80-GeV Ar40 Ions. Physical Review C, 17, 1632. http://dx.doi.org/10.1103/PhysRevC.17.1632

[4] Kaufman, S. and Steinberg, E. (1980) Cross-Section Measurements of Nuclides Formed by the Reaction of 0.20-6.0 GeV Protons with Au197. Physical Review C, 22, 167. http://dx.doi.org/10.1103/PhysRevC.22.167

[5] Fortney, D. and Porile, N. (1980) Angular Distributions of Sc Fragments from the Interaction of U238 with 0.8-400 GeV Protons. Physical Review C, 21, 2511. http://dx.doi.org/10.1103/PhysRevC.21.2511

[6] Sandoval, A., Gutbrod, H., Meyer, W., et al. (1980) Spectra of p, d, and t from Relativistic Nuclear Collisions. Physical Review C, 21, 1321. http://dx.doi.org/10.1103/PhysRevC.21.1321

[7] Danielewicz, P. and Pan, O. (1992) Blast of Light Fragments from Central Heavy-Ion Collisions. Physical Review C, 46, 2002. http://dx.doi.org/10.1103/PhysRevC.46.2002

[8] Alba, R., Coniglione, R., Del Zoppo, A., et al. (1994) On the Origin of Fast Proton Emission in Intermediate Energy Heavy Ion Collisions. Physics Letters B, 322, 38-42. http://dx.doi.org/10.1016/0370-2693(94)90488-X

[9] Chen, K., Fraenkel, Z., Friedlander, G., et al. (1968) VEGAS: A Monte Carlo Simulation of Intranuclear Cascades. Physical Review, 166, 949. http://dx.doi.org/10.1103/PhysRev.166.949

[10] Amsden, A.A., Goldhaber, A.S., Harlow, F.H. and Rayford, N.J. (1978) Relativistic Two-Fluid Model of NucleusNucleus Collisions. Physical Review C, 17, 2080-2096. http://dx.doi.org/10.1103/PhysRevC.17.2080

[11] Izumo, K. and Araseki, H. (1983) Time Compound Nucleus for High Energy Nuclear Reactions. Progress of Theoretical Physics, 69, 158-170. http://dx.doi.org/10.1143/PTP.69.158

[12] Goto, T., Yamaguchi, K. and Naka, S. (1980) The Time as an Observable in Quantum Mechanics. Progress of Theoretical Physics, 64, 1-17. http://dx.doi.org/10.1143/PTP.64.1

[13] Olkhovsky, V.S. (1984) Investigation of Nuclear Reactions and Decays by Analysis of Their Duration. Soviet Journal of Particles and Nuclei, 15, 130-148.

[14] Lyuboshitz, V.L. (1977) On the Collision Duration in the Presence of Strong Overlapping Resonamce Levels. Physics Letters B, 72, 41-44. 
[15] Lyuboshitz, V.L. (1978) On Duration of Nuclear Reactions under Conditions of the Strong Overlapping of the Resonance Levels. Soviet Journal of Nuclear Physics, 27, 948-955.

[16] Lyuboshitz, V.L. (1978) Unitary Sum Rules and the Collision Time under the Strong Overlapping of the Resonance Levels. JETP Letters, 28, 32-37.

[17] D’Arrigo, A., Giardina, G., Taccone, A., Hassani, G. and Olkhovsky, V. (1993) A Way to Evaluate the Level Density, Total Width and Compound-Nucleus Decay Functions in the Range of Unresolved Resonances. International Journal of Modern Physics E, 2, 809-820.

[18] Olkhovsky, V.S. and Omelchenko, S.A. (1996) Joint Time-Evolution and Statistical Energy-Resonance Analysis of High-Energy Nuclear Collisions and Possible Phenomena of Time Resonances. In: Giardina, G., Fazio, G. and Lattuada, M., Eds., Brolo, 15-19 October 1996, World Scientific, 410-420.

[19] Olkhovsky, V.S., Dolinska, M.E. and Omelchenko, S.A. (2012) On the Cross Section and Duration of the NeutronNucleus Scattering with a Resonance, Distorted by a Non-Resonant Background, in the Center-of-Mass System and Laboratory System. Proceedings of the 4th International Conference on Current Problems in Nuclear Physics and Atomic Energy, Kyiv, 3-7 September 2012, 198-201.

[20] Olkhovsky, V.S., Dolinska, M.E. and Omelchenko, S.A. (2009) Phenomena of Time Resonances Explosions for the Compound-Clot Decays in High-Energy Nuclear Reactions. 15 p.

[21] Prilepsky, Y.B. and Shmonin, V.L. (1987) On the Mechanism of Cumulative Proton and Nuclear Fragment Production. Acta Physica Polonica B, 18, 729.

[22] Simonius, M. (1974) Overlapping Resonances and Unitarity of the Scattering Matrix. Nuclear Physics A, 218, 53-60. http://dx.doi.org/10.1016/0375-9474(74)90019-0

[23] Baz, A.I. (1978) Lifetime of Strongly Excited Nuclei. JETP Letters, 27, 132-135. 